\title{
An unusual retroperitoneal lesion causing recurrent acute pancreatitis
}

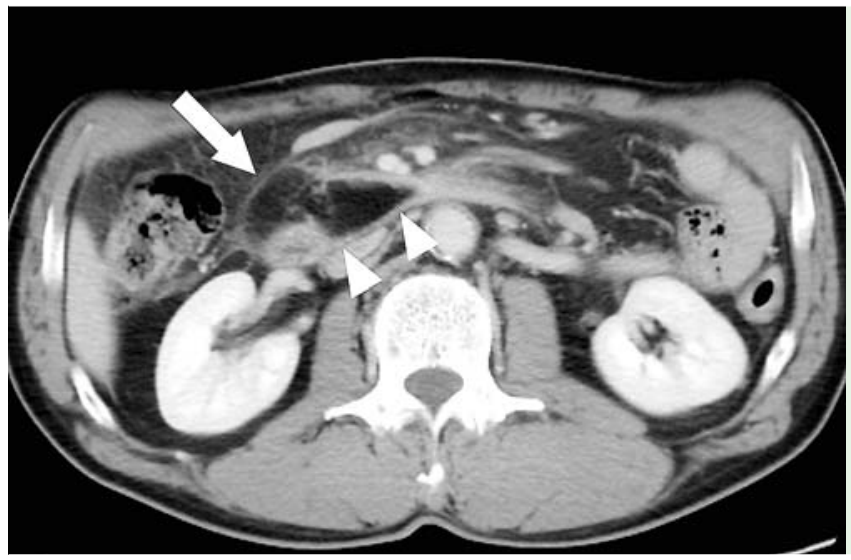

Fig. 1 Abdominal computed tomographic scan shows an encapsulated mass of fatty density (arrow) compressing the third portion of the duodenum (arrowheads) in a 71-year-old man presenting with postprandial upper abdominal pain.

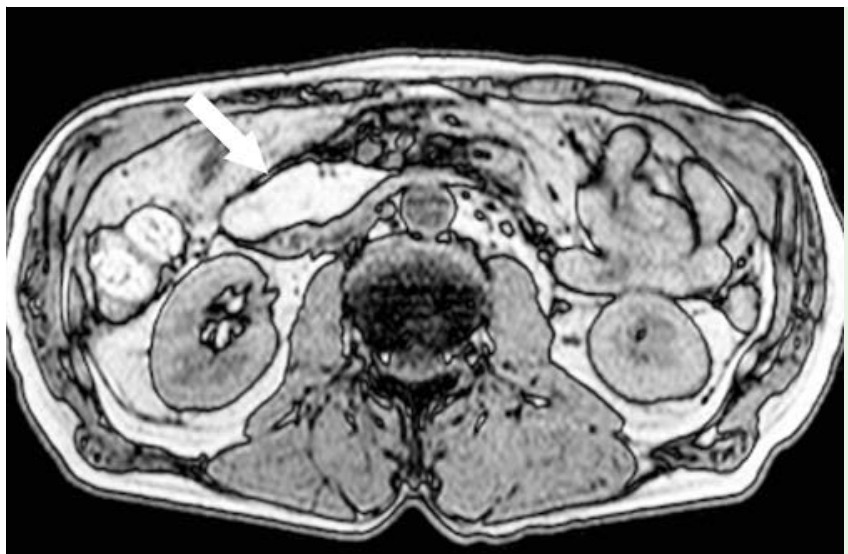

Fig. 2 Axial T1-weighted magnetic resonance image shows a well-demarcated, hyperintense mass (arrow).

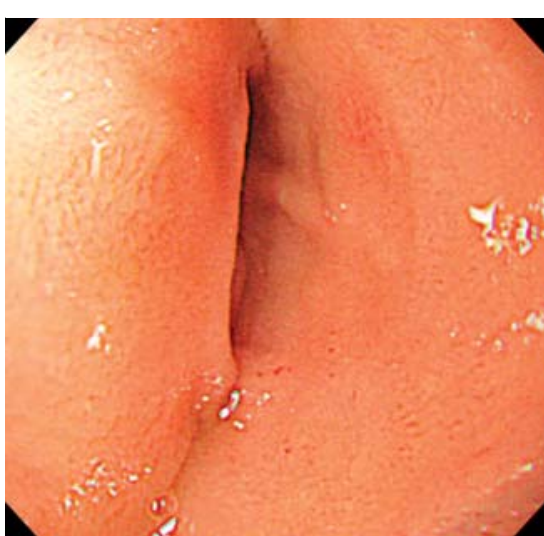

Fig. 3 Endoscopic of view of the third portion of the duodenum with luminal narrowing.

A 71-year-old man presented with postprandial upper abdominal pain. He had not drunk any alcohol. Nevertheless, he had experienced four episodes of acute pancreatitis of unknown etiology in the last 2 months. His serum amylase (3135U/L) and lipase (8472U/L) were elevated, and subsequent computed

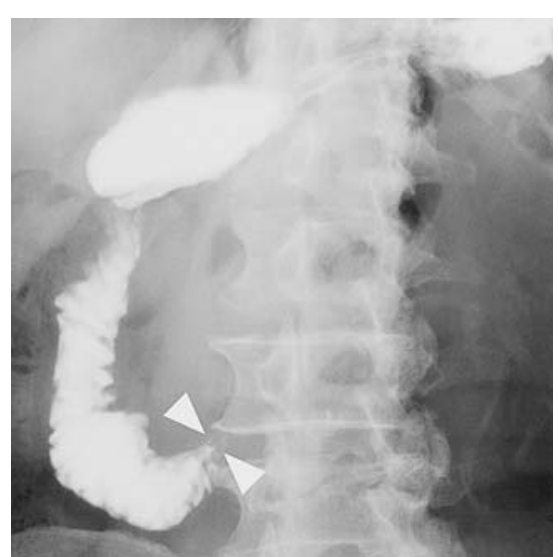

Fig.4 Gastroduodenography shows duodenal narrowing in the third portion (arrowheads).

tomography showed acute pancreatitis and an encapsulated mass of fatty density around the duodenum ( $\bullet$ Fig. 1 ).

The mass was depicted more clearly by magnetic resonance (MR) imaging and appeared as a well-demarcated, homogeneous, hyperintense lesion on T1-weighted images ( $\bullet$ Fig.2). Endoscopy and gas- troduodenography demonstrated duodenal narrowing in the third portion due to extrinsic compression ( Fig. 3 and - Fig.4). MR cholangiopancreatography, endoscopic ultrasound, and endoscopic retrograde cholangiopancreatography revealed no definite cause of the acute pancreatitis; there was no evidence of choledocholithiasis, pancreatic tumor, or pancreaticobiliary malformation. The imaging findings suggested that the third portion of the duodenum was being compressed by a retroperitoneal lipoma. Thus, the cause of the recurrent acute pancreatitis was presumed to be a transient increase in duodenal pressure.

The retroperitoneal mass was resected surgically, and the entire specimen showed features consistent with a retroperitoneal lipoma. No pancreatitis has been observed for 20 months.

Recurrent acute pancreatitis results most frequently from alcohol, followed by gallstones [1]. Increased duodenal pressure, which occurs as a result of superior mesenteric artery syndrome or afferent loop syndrome, can also be a cause of recurrent acute pancreatitis [2,3]. Retroperitoneal lipoma is a rare condition that presents as a large mass causing abdominal swelling or symptoms due to the obstruction of adjacent organs [4]. MR imaging is the modality of choice for imaging lipoma; it is depicted as a discrete, encapsulated, homogeneous fatty mass [5]. To the best of our knowledge, no similar case has been reported in the literature.

Endoscopy_UCTN_Code_CCL_1AZ_2AB

Competing interests: None

\section{Sho Kitagawa, Hiroyuki Miyakawa}

Department of Gastroenterology, Sapporo Kosei General Hospital, Sapporo, Japan

\section{References}

1 Gullo L, Migliori M, Pezzilli R et al. An update on recurrent acute pancreatitis: data from five European countries. Am J Gastroenterol 2002; 97: 1959-1962

2 Sihuay-Diburga DJ, Accarino-Garaventa A, Vilaseca-Montplet $J$ et al. Acute pancreatitis and superior mesenteric artery syndrome. Rev Esp Enferm Dig 2013; 105: 626-628

3 Mithöfer K, Warshaw AL. Recurrent acute pancreatitis caused by afferent loop stricture after gastrectomy. Arch Surg 1996; 131: $561-565$

4 Weniger M, D'Haese JG, Kunz W et al. En-bloc resection of a giant retroperitoneal lipoma: a case report and review of the literature. BMC Res Notes 2015; 8: 75 
5 Gaskin CM, Helms CA. Lipomas, lipoma variants, and well-differentiated liposarcomas (atypical lipomas): results of MRI evaluations of 126 consecutive fatty masses. AJR Am J Roentgenol 2004; 182: 733-739

\section{Bibliography}

Dol http://dx.doi.org/

10.1055/s-0034-1392563

Endoscopy 2015; 47: E393-E394

(c) Georg Thieme Verlag KG

Stuttgart · New York

ISSN 0013-726X
Corresponding author

\section{Sho Kitagawa, MD}

Department of Gastroenterology Sapporo Kosei General Hospital Kita 3 Higashi 8

Chuo-ku

Sapporo 060-0033

Japan

Fax: +81-11-271-5320

bossa0405@yahoo.co.jp 